\title{
Adenofibroma mülleriano tratado con análogos de GnRH. Reporte de un caso y revisión de la literatura
}

\author{
Juan Carlos Sabogal M.D.*; Lilia Sánchez M.D.**; Jaime Ferro M.D.****
}

\begin{abstract}
RESUMEN
Se presenta el caso de una paciente de 29 años quién acudió a nuestra consulta por sensación de masa en hipogastrio. El examen físico evidenció un útero aumentado de tamaño y la ecografía mostró la presencia de una masa intrauterina interpretada inicialmente como un mioma intracavitario por lo cual la paciente recibió tratamiento preoperatorio con Acetato de Leuprolide, observándose desaparición de la masa. Se realizó una histerosonografía que reveló una imagen polipoide endometrial por lo que la paciente se llevó a legrado. A los tres meses de seguimiento, se observó nuevamente aumento del tamaño uterino. La revisión de la patología permitió hacer el diagnóstico de un Adenofibroma Mülleriano de lo que inicialmente se interpretó patológicamente como un pólipo endometrial. La paciente fue llevada a histeroscopia operatoria previa administración de otro ciclo de Leuprolide, con óptimo pronóstico obstétrico y permaneciendo libre de recidiva tumoral 6 meses después. Se considera que resulta novedosa la respuesta de éste tipo tumoral a los análogos de la GnRH y que el abordaje histeroscópico mejora la posibilidad de manejo conservador que antes era difícil.
\end{abstract}

PALABRAS CLAVES: Adenofibroma Mülleriano, Análogos de GnRH, Histeroscopia, Tumores uterinos.

\section{SUMMARY}

We present the case of a 29 years old woman complaining about abdominal mass. Physical exam showed an enlarged uterus and ultrasound revealed an intrauterine mass thought to be an endometrial mioma. Planning miomectomy, patient received 3 months treatment with Leuprolide Acetate after which, the lesion disappeared. Histerosonography showed a residual polipoid endometrial lesion and the patient was taken to curettage. After 3 months, follow-up examination showed an enlarged uterus and pathology material was reexamined and the former endometrial polyp turned to be a Müllerian Adenofibroma. Then, due to the previous response to medication, another 3 months of Leuprolide were administered and histeroscopic resection was then performed with optimal obstetrical prognosis and remaining free of tumor after 6 months follow-up. There are not previous reports in literature about the effect of GnRH on this kind of neoplasm and we feel its use combined with histeroscopy, improves the possibility of conservative management in selected cases.

KEY WORDS: Müllerian Adenofibroma, GnRH Analogues, Histeroscopy, Uterine Tumors.

\section{Introducción}

El Adenofibroma Mülleriano (AFM) es un tumor de rara presentación, de origen mixto constituido por células epiteliales y tejido conectivo que suele comprometer el útero. Aunque histológicamente es benigno, su comportamiento biológico suele ser agresivo debido a su carácter multifocal y a las recidivas propias de su historia natural. Como consecuencia, el tratamiento de esta lesión en pacientes con paridad satisfecha ha sido la histerectomía y en pacientes nulíparas el tratamiento es motivo de controversia. Deseamos presentar el caso de una paciente con ésta entidad que se benefició de la

Profesor Asistente, Departamento de Ginecología y Obstetricia. Universidad Nacional de Colombia. Instituto Materno Intantil. Bogotá.

Profesora Asistente. Departamento de Patología. Universidad Nacional de Colombia. Instituto Materno Infantil. Bogotí.

Profesor Asociado, Departamento de Ginecología y Obstetricia. Universidad del Rosario. CECOLFES, Bogotá. administración de análogos de la GnRH y posterior resección histeroscópica, con óptimo pronóstico.

\section{Caso clínico}

Se trata de una paciente de raza mestiza, casada, de 29 años que consultó al servicio de ginecología del Instituto Materno Infantil en agosto de 1997, por sensación de masa en hipogastrio de 4 meses de evolución, indolora y de crecimiento progresivo, asociado a la presencia de ciclos menstruales irregulares de $10 \times 3$ en los últimos 6 meses. Los antecedentes quirúrgicos, médicos y tóxico-alérgicos eran irrelevantes y dentro de los antecedentes ginecológicos, menarquia a los 14 años, con ciclos regulares de 30 X 4 hasta 6 meses antes de la consulta. G0P0, planificación (-), sexualmente activa y con deseo de embarazo. El examen físico reveló una paciente en buenas condiciones generales, que como datos positivos presentó una masa de $15 \times 15 \mathrm{~cm}$ en hipogastrio, ocupando la línea media, de superficie lisa, consistencia dura, adherida a planos profundos, no dolorosa y simétrica. Al tacto vaginal, una 
vagina normal, con un cuello duro, posterior, cerrado y un útero aumentado uniformemente de tamaño, también duro. con una longitud de $16 \times 12 \times 12 \mathrm{~cm}$, anexos uterinos (-), sin masas. Se hizo un diagnóstico de miomatosis uterina y la paciente fue enviada a ecografía que reportó: "mioma intracavitario" (figura 1). Se le propuso a la paciente una miomectomia y se administró un curso intramuscular de Acetato de Leuprolide de $3.75 \mathrm{mg} /$ mes por 3 meses, previo al procedimiento quirúrgico. En el control preoperatorio, se evidenció inesperadamente, un útero de tamaño, forma y consistencia normales. Ante la "ausencia" del mioma, se practicó una histerosonografía, que evidenció una lesión residual de aspecto polipoide de $2 \mathrm{x}$ $4 \mathrm{Cm}$, dependiente de la cara lateral derecha de la cavidad endometrial (figura 2) y con diagnóstico de pólipo endometrial se llevó a legrado, obteniéndose material de aspecto polipoide que se envió a estudio anátomo-patológico, e inicialmente reportado como: "Pólipo endometrial", por lo cual se le dio de alta sin ulterior tratamiento, reapareciendo 3 meses después a control evidenciándose nuevamente crecimiento uterino, a pesar de que la paciente permaneció asintomática, con menstruación en el último mes. Debido al carácter recidivante de la lesión, se solicitó reexaminar la patología que fue reportada ésta vez como: "Adenofibroma Mülleriano" (figura 3). En vista de la nuliparidad de la paciente y de la respuesta del tumor a un primer ciclo con Acetato de Leuprolide, se administró un segundo ciclo similar al anterior (también de 3 meses), seguido de resección histeroscópica en el consultorio y bajo anestesia local durante la cual, se hizo visualización directa de una lesión polipoide que comprometía el aspecto lateral derecho de la pared endometrial posterior, compuesta por múltiples prolongaciones digitiformes. arracimadas y que protruían hacia la cavidad endometrial. Se corroboró la presencia de otro foco polipoide en la línea media de la pared endometrial posterior de caracte rísticas similares a la primera lesión descrita, aunque de menor tamaño. Mediante resectoscopio, se realizó ex-

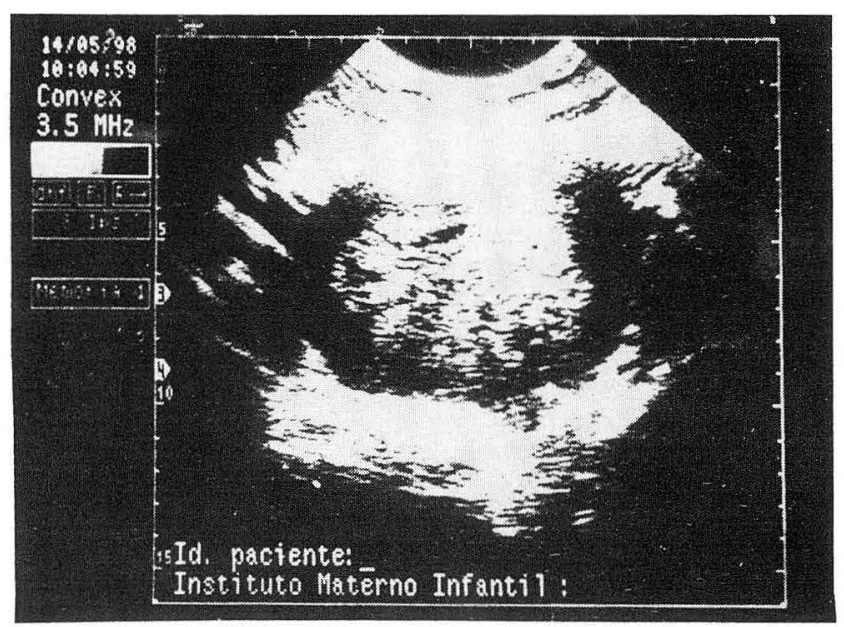

Figura $0010 \mathrm{i0l}$

Ultrasonido inicial. Se observa una masa sólida con pequeñas áreas quísticas que ocupa toda la cavidad uterina que media $10 \times 10 \mathrm{~cm}$. tracción de las lesiones realizando un corte más basal. El procedimiento, realizado ambulatoriamente, tuvo una evolución satisfactoria sin complicaciones. Un mes después, la paciente fue citada para histeroscopia de control que mostró una cavidad sin lesión remanente. El seguimiento realizado con histeroscopia bimensual, al cabo de seis meses no mostró recidiva tumoral. En el momento, la paciente se encuentra bajo control médico por la consulta de infertilidad.

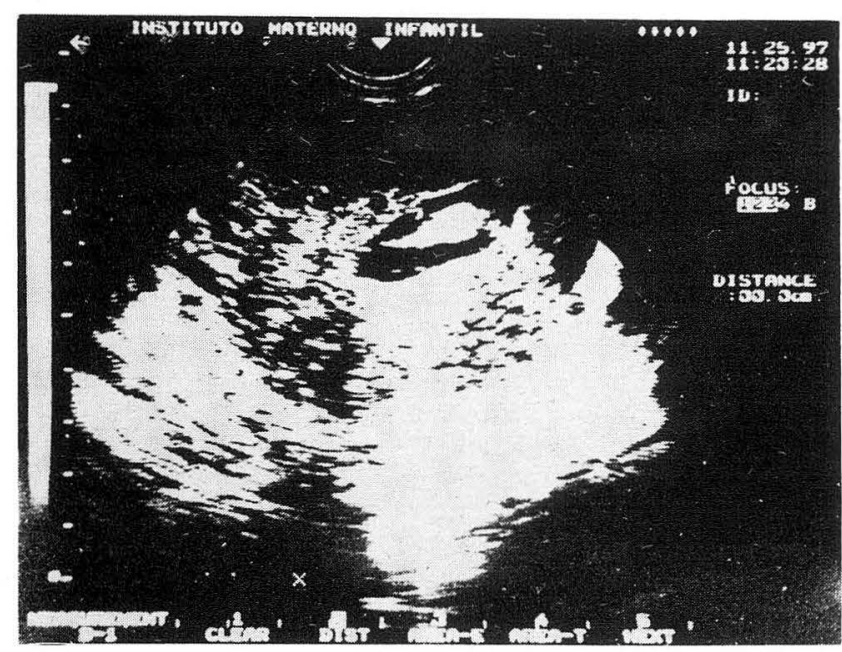

Figura $0010 \mathrm{i02}$

Histerosonografía luego de 3 meses de Leuprolide. Se observa una lesión residual pediculada de 4 X 2 cms., dependiente de la cara lateral derecha de la cavidad endometrial.

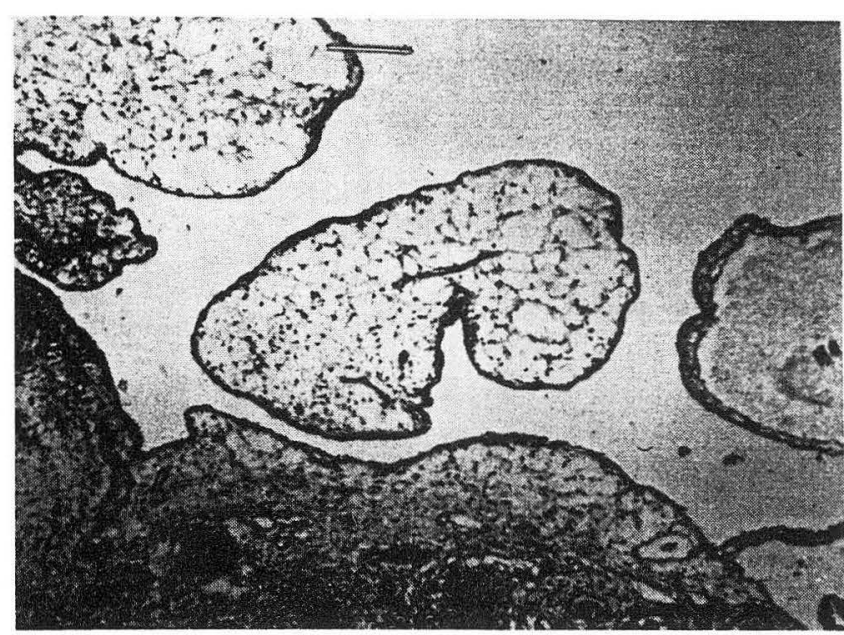

Figura 0010i03

Aspecto histológico de la lesión. Se observan formaciones digitiformes cortadas transversalmente compuestas por un epitelio de revestimiento simple y un estroma edematoso, laxo, poco celular y con algunos vasos sanguíneos, características propias del adenofibroma Mülleriano. 


\section{Patología}

En el laboratorio de Patología se recibieron inicialmente múltiples fragmentos irregulares de tejido pardo que en conjunto hacían un volumen de 3 c.c; éste material fue interpretado como un pólipo endometrial. Sin embargo, dado el comportamiento biológico de la lesión, la lámina histológica fue revisada nuevamente destacándose la presencia de papilas romas revestidas por un epitelio cúbico simple sin atipias, algunas con un tallo constituido por estroma endometrial poco celular y tejido fibroconectivo y otras con un estroma francamente edematoso (figura 3), sin actividad mitótica. Este aspecto característico llevó a concluir la presencia de un AFM. Unos meses después se recibió el material obtenido durante la histeroscopia siendo las características del material similares a las descritas previamente.

\section{Discusión}

Por definición, el AFM es un tumor benigno, raro, del cual se han reportado en la literatura sólo 40 casos (4-5), muchos de los cuales han ocurrido en mujeres postmenopáusicas con un promedio de edad de 68 años; sin embargo la distribución por edades es amplia reportándose casos entre los 19 y 80 años. La forma más frecuente de presentación es el sangrado uterino anormal, aunque algunos pacientes consultan por dolor. En el examen pélvico se encuentra el útero aumentado de tamaño y en algunas ocasiones se pueden observar masas polipoides protruyendo a través del cuello uterino. Solamente una pequeña cantidad de casos ha sido diagnosticada por medio del curetaje endometrial antes de que se haya realizado la histerectomía, como es el caso de nuestra paciente. Estas neoplasias tienen diámetros que varían de 2 a $20 \mathrm{~cm}$ y macroscópicamente son de crecimiento exofítico sobre una base amplia dentro de la cavidad endometrial. Aunque se consideran benignos, tienden a presentar recurrencias locales después de que son resecados incompletamente y por tanto el tratamiento que se recomienda es la histerectomía (1).

En la actualidad la experiencia que existente para el diagnostico del AFM en material de biopsia es limitada. Se podría esperar que el material para estudio histopatológico fuera abundante, con algunos fragmentos que muestren papilas que se ramifican y que tienen un centro fibroso. También sería evidente la variación marcada entre uno y otro fragmento en los cuales se podría ver fibrosis del estroma o quistificación y predominio de glándulas dilatadas. Las Papilas están formadas por células pequeñas del estroma endometrial o fibroblastos, siempre acompañados de abundante colágeno. El epitelio de revestimiento puede ser cúbico o cilíndrico, con o sin estratificación y atipia citológica. Usualmente estas células son de tipo endometrial, sin embargo se han visto también en el revestimiento células mucinosas, tubáricas y epitelio escamoso. La actividad mitótica es rara. Como criterio para diferenciar el AFM de su contraparte maligna se utiliza el índice mitótico: el hallazgo de menos de 2 a 4 mitosis por 10 campos de gran aumento orienta hacia el AFM $(2,3)$. Otro hallazgo que permite diferenciar estas dos lesiones es la mayor celularidad del estroma y la tendencia a formar cúmulos de células glandulares. $\mathrm{Al}$ igual que con el adenosarcoma los diagnósticos diferenciales desde el punto de vista histológico del AFM son el pólipo endometrial, el cual siempre muestra un estroma colágeno y glándulas inactivas. Sin embargo, la diferenciación entre esta lesión y el AFM puede estar en que este último no presenta papilas de superficie amplia (1).

Nuestra paciente es una mujer joven, sin embarazos previos que por lo inusual de su lesión, se prolongó en su proceso diagnóstico. Como se ha descrito antes, habitualmente ésta neoplasia suele ser confundida con otras entidades y sólo la historia natural de recidivas inesperadas orienta hacia el diagnóstico correcto(2). Según se ha descrito, éste tumor puede aparecer en épocas relativamente tardías de la vida en las cuales el futuro obstétrico no es importante y por lo tanto la histerectomía como tratamiento definitivo no es objetada por la paciente. En éste caso, la cirugía radical (histerectomía), no procedía debido a la nuliparidad de la paciente. A pesar de que el tratamiento con el Leuprolide fue iniciado con otro diagnóstico, la respuesta dramática del tumor resultó muy conveniente para las necesidades de la paciente. Dicha respuesta no ha sido descrita como tratamiento habitual del adenofibroma y evidentemente, representa gran beneficio en aquellos casos de pacientes jóvenes afectadas por este tumor en las cuales la conservación de la función reproductiva es fundamental. La acción de la medicación sobre este tumor sugiere que existe una influencia hormonal sobre el crecimiento de la lesión, característica que tampoco ha sido mencionada en la literatura. Es curioso que a pesar de tal influencia, en ocasiones el tumor está haciendo su aparición en épocas tardías de la vida, luego de muchos años de actividad hormonal sobre el tejido potencialmete neoplásico que podría asumirse es un remanente embriológico según algunas teorías (3). A pesar de que el curso farmacológico fue corto (tres meses), es posible que se obtengan resultados igualmente satisfactorios con tiempos menores, asumiendo que la dependencia hormonal del tumor en cuestión sea notoria. Es importante señalar, que el abordaje histeroscópico probablemente sea determinante en el pronóstico reproductivo de la entidad ya que es un procedimiento rápido que puede realizarse en el consultorio bajo anestesia local, es comparativamente barato y en manos entrenadas es seguro y eficaz. En el futuro, la disponibilidad de mejores instrumentos para la resección histeroscópica logrará disminuir la recidiva tumoral. En conclusión, el caso presentado plantea la posibilidad de un nuevo tratamiento más conservador para las pacientes jóvenes afectadas por el Adenofibroma Mülleriano, permitiendo un futuro reproductivo que antes era imposible. 


\section{BIBLIOGRAFIA}

1. Zaino RJ. Interpretation of Endometrial Biopsies and Curettings. Biopsy Interpretation series. Lippincott-Raven. 1996; Pág. 338-340.

\section{FORMULA:}

PREMELLE GRAGEAS, PREMELLE PLUS, PREMELLE CICLICO incluye: estrógenos conjugados $0,625 \mathrm{mg} y$ acetato de medroxiprogesterona (MPA), 2,5 mg o $5,0 \mathrm{mg}$, para administración oral. PREMELLE GRAGEAS, PREMELLE PLUS Y PREMELLE CICLICO contiene una mezcla de estrógenos obtenidos exclusivamente de fuentes naturales, están compuestos por las sales sódicas de ésteres sulfato hidrosolubles de la estrona, la equilina y la 17 alfa-hidroxiequilina, además de cantidades más pequeñas de 17 alfa-estradiol, equilenina, 17-alfa-dihidroequilenina, 17 beta-hidroequilina, 17 beta-dihidro- equilenina, 17 beta-estradiol y delta 8,9dehidroestrona.

\section{INDICACIONES:}

1. Sintomas vasomotores moderados a severos asociados con una deficiencia de estrógenos.

2. Prevención y manejo de la Osteoporosis asociada con una deficiencia de estrógenos.

3. Vaginitis y Uretritis atrófica.

PREMELLE GRAGEAS, PREMELLE PLUS Y PREMELLE CíCLICO se encuentra indicado en mujeres con útero intacto a fin de prevenir el riesgo de hiperplasia endometrial y cáncer endometrial asociado con la terapia de suplencia estrógenica. En el caso de pacientes histerectomizadas la sola terapia de Premarin es apropiada.

\section{CONTRAINDICACIONES:}

1. Cáncer conocido o sospechado de las mamas.

2. Neoplasia conocida o sospechada de estrógeno-dependiente

3. Embarazo conocido o sospechado

4. Hemorragia genital anormal no diagnosticada

5. Trastornos tromboflebíticos o tromboembólicos

6. Disfunción o enfermedad hepática

7. Hipersensibilidad a cualquiera de los componentes de PREMELIE GRAGEAS, PREMELLE PLUS Y PREMELLECICLICO

POSOLOgía Y ADMINISTRACIón: La terapia continua con PREMELLE GRAGEAS, PREMELLE PLUS consiste en $0,625 \mathrm{mg}$ de estrógenos conjugados y $2,5 \mathrm{mg} 05 \mathrm{mg}$ de acetato de medroxiprogesterona para ser tomados simultáneamente una vez al día. La terapia continua con PREMELLE GRAGEAS, PREMELLE PLUS está indicada en mujeres con útero intacto para reducir el riesgo de hiperplasia endometrial y cáncer endometrial asociado con la terapia de suplencia estrogénica. La terapia sólo con Premarin solamente es apropiada para las pacientes histerectomizadas. La dosis inicial de 5,0 mg de acetato medroxiprogesterona es apropiada para aquellas pacientes en las cuales se deban lograr ciclos sin sangrado. Considérense la reducción de la dosis de $2.5 \mathrm{mg}$ después de alcanzar la amenorrea o dentro de los 12 meses de iniciada la terapia. Si un sangrado ocurre después de la reducción del acetato de medroxiprogesterona a $2,5 \mathrm{mg}$ considere reiniciar el régimen de $5,0 \mathrm{mg}$. La dosis inicial habitual con PREMELLE CONTINUO, Y PREMELLE CíCLICO es de $5,0 \mathrm{mg}$.

Adicionalmente se deben tener en cuenta los siguientes factores cuando se ajuste la dosis del acetato de medroxiprogesterona: historia médica de la paciente, presencia de eventos adversos, resultado de laboratorio y exámenes fisico y ginecológico. Las pacientes deben ser evaluadas nuevamente en intervalos regulares.

\section{ADVERTENCIAS:}

Algunos estudios sugirieron un posible aumento de la incidencia de cáncer de mama en aquellas mujeres que reciben dosis elevadas de estrógenos durante un periodo prolongado. Sin embargo, la mayoría de los estudios no corroboraron esta asociación con las dosis habitualmente utilizadas para la terapia de suplencia estrogénica. Las mujeres que reciben este tratamiento deben ser evaluadas periódicamente con exámenes mamarios y recibir instrucciones para que puedan llevar a cabo el autoexamen mamario. Se reportó un riesgo aumentado de enfermedad de la vesícula biliar en las mujeres posmenopáusicas tratadas con estrógenos. Las dosis de PREMELLE GRAGEAS, PREMELLE PLUS Y PREMELLE CICLICO usadas en la terapia de suplencia hormonal no deben ser mayores que las dosis recomendadas. El uso de PREMELLE GRAGEAS, PREMELLE PLUS Y PREMELLE CÍCLICO durante la menopausia se asoció con aumentos de la presión sanguínea. Se recomienda el control periódico de la presión arterial.

No se documentó un riesgo aumentado de tromboflebitis o de accidentes tromboembólicos en las mujeres que reciben una terapia de suplencia estrogénica. Sin embargo, no se cuenta con una cantidad suficiente de información para establecer con certeza dicho riesgo en las mujeres con antecedentes de enfermedad tromboembólica. El médico debe mantenerse alerta a fin de detectar las manifestaciones más tempranas de los trastornos trombóticos (tromboflebitis, accidentes cerebrovasculares, embolia pulmonar y trombosis retiniana). Ante la aparición o la sospecha de cualquiera de estos problemas se encuentra indicada la interrupción inmediata de PREMELLE GRAGEAS, PREMELLE PLUS Y PREMELLE CÍCLICO y suministrar tratamiento apropiado a la paciente.

Se recomienda interurmpir el tratamiento con PREMELLE GRAGEAS, PREMELLE PLUS Y PREMELLECÍCLICO hasta esperar los resultados del examen médico ante la aparición de una pérdida parcial o completa de la visión o ante la instalación brusca de proptosis, diplopia o migraña. Si el examen oftalmológico revela edema de papila o lesiones vasculares retinianas, el tratamiento debe ser definitivamente interrumpido.

USO DURANTE EL EMBARAZO: PREMELLE GRAGEAS, PREMELLEPLUS Y PREMELLE CÍCLICO no deben ser utilizados durante el embarazo.

\section{PRECAUCIONES:}

Es importante obtener una información médica y familiar completa antes de iniciar cual quier tratamiento con estrógenos. Loe exámenes físicos pretratamiento y periódicos durante el tratamiento deben consignar cualquier dato anormal relacionado con la presión sanguínea, las mamas, el abdomeny los pelvianos e incluir un extendido con la técnica de Papanicolaou.

\section{PRESENTACIONES:}

PREMELLE GRAGEAS: Caja × 28 grageas de Estrógeno Naturales Conjugados $0,625 \mathrm{mg}$ y Acetato de Medroxiprogesterona 2,5 mg Reg. INVIMAM-010941.

PREMELLE PLUS Grageas: Caja de 28 grageas de Estrógenos Naturales Conjugados 0,625 mg y Acetato de Medroxiprogesterona 5,0 mg Reg. INVIMAM-010940.

PREMELLE CICLICO: Caja x 28 grageas: 14 grageas de Estrógenos Naturales Conjugados 0,625 mg y 14 grageas de Estrógenos Naturales Conjugados $0,625 \mathrm{mg}+$ Acetato de Medroxiprogesterona $5 \mathrm{mg}$. Reg. INVIMA M-011988

\section{REFERENCIAS:}

1. Archer DF, Pickar JH, Bottiglioni F for The Menopause Study Group. Obstetric Gynecol 83:686-692

2. Woodruff JD, Pickar JH,for The Menopause Study Group, Am J Obstetric Gynecol 70:1213-1223, 1994
2. Zaloudek C., Norris H. Adenofibroma and adenosarcoma of the uterus: a clinicopathologic study of 35 cases. Cancer 1981; 48: 354-366.

3. Clement P.. Scully R. Tumors with mixed epithelial and mesenchymal elements. In: Clement P, Young R, eds. Tumors and tumorlike lesions of the uterine corpus and cervix. New York: Churchill Livingstone; 1993; 329-369 (Roth LM,ed; Contemporary issues in surgical pathology; vol 19).

4. Vellios F. Papillary adenofibroma-adenosarcoma: the uterino cystosarcoma phylodes. In Fenoglio C, Wolff M, eds. Progress in surgical pathology. New York: Masson; 1980; (1): 205-219.

5. Clement PB, Scully RE. Múllerina adenofibroma of the uterus with invasion of myometrium an pelvic veins. Int J. Gynecol Pathol 1990; 9(4): $363-371$.

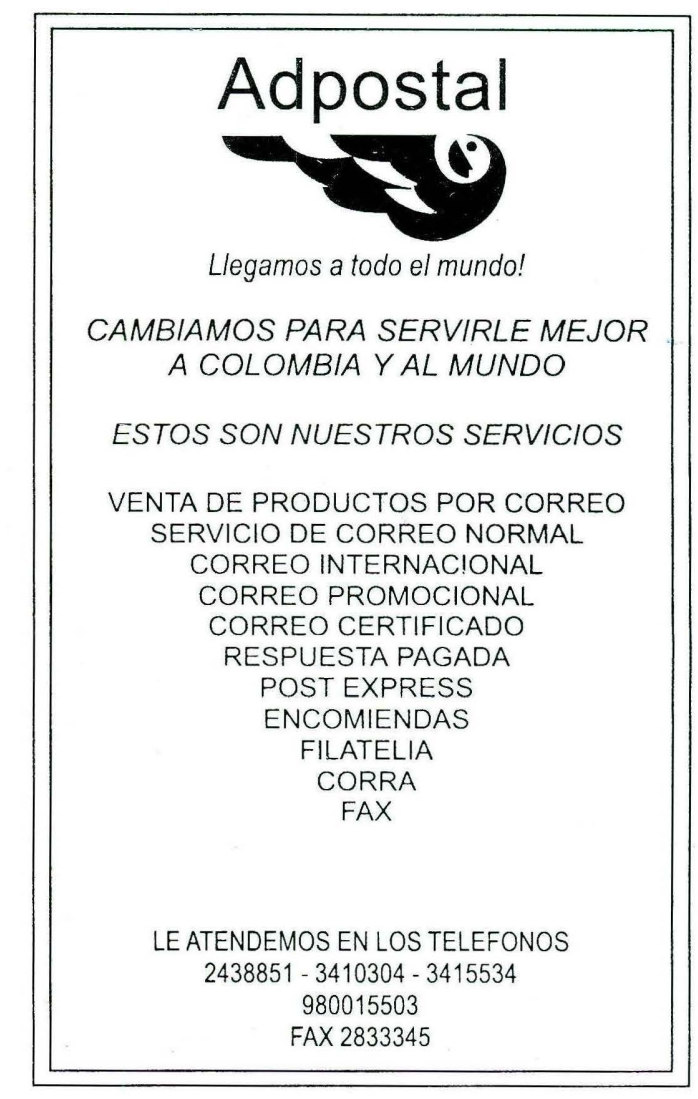

